\title{
Stem cells in the adult rat spinal cord: plasticity after injury and treadmill training exercise
}

\author{
Ariane Foret, ${ }^{, 1}$ Renaud Quertainmont, ${ }^{*}, 1$ Olivier Botman, ${ }^{*}$ Delphine Bouhy, ${ }^{*}$ Philippe Amabili, ${ }^{*}$ \\ Gary Brook, $\dagger$ Jean Schoenen* + and Rachelle Franzen* \\ * GIGA Neurosciences, University of Liege, Liege, Belgium \\ $\dagger$ Department of Neuropathology, University of Aachen, Aachen, Germany \\ $\$$ Department of Neurology, University of Liege, Liege, Belgium
}

\begin{abstract}
Ependymal cells located around the central canal of the adult spinal cord are considered as a source of neural stem cells (NSCs) and represent an interesting pool of endogenous stem cells for repair strategies. Physical exercise is known to increase ependymal cell proliferation, while improving functional recovery. In this work, we further characterized those endogenous NSCs within the normal and injured adult rat spinal cord and investigated the effects of treadmill training using immunohistochemical and behavioral studies. In uninjured untrained rats, Sox-2, a NSC marker, was detected in all ependymal cells of the central canal, and also scattered
\end{abstract}

throughout the parenchyma of the spinal cord. Within the lesion, Sox-2 expression increased transiently, while the number of nestin-positive ependymal cells increased with a concomitant enhancement of proliferation, as indicated by the mitotic markers Ki67 and bromo-deoxyuridine. Exercise, which improved functional recovery and autonomous micturition, maintained nestin expression in both injured and uninjured spinal cords, with a positive correlation between locomotor recovery and the number of nestin-positive cells. Keywords: ependyma, functional recovery, neural stem cell, Sox-2, spinal cord injury, treadmill training.

J. Neurochem. (2010) 112, 762-772.
The adult mammalian CNS has long been claimed to be unable to repair itself after injury (Ramon Cajal 1928). The discovery of neural stem cells (NSCs) in the adult brain (Reynolds and Weiss 1992) led to the re-evaluation of this dogma. It raised new hopes for the development of stem cellbased reparative therapies, either by transplantation or by recruitment of endogenous NSC. In the intact adult spinal cord, NSCs have been reported to be quiescent and located in the ependymal layer or the sub-ependymal zone of the central canal (Weiss et al. 1996; Johansson et al. 1999; Shihabuddin 2008). These cells, after in vitro expansion, can form neurospheres and be transplanted into adult rat spinal cord injury (SCI), where they promote functional recovery (Parr et al. 2007). Following experimental SCI, adult NSCs have been reported to proliferate and migrate towards the lesion site, where they differentiated into astrocytes, effectively contributing to glial scar formation (Johansson et al. 1999). Recently, oligodendrogenesis and neurogenesis have been observed in the injured spinal cord following the administration of basic fibroblast growth factor and epidermal growth factor directly into the injured tissue (Ohori et al. 2006). In addition to their potential in cell replacement strategies, NSCs demonstrate the capacity to affect a wide range of recovery processes. For example, following transplantation into the injured spinal cord, NSCs have been reported to express growth factors such as brain-derived neurotrophic factor (BDNF), nerve growth factor, and glial cell line-derived neurotrophic factor, which generated a beneficial microenvironment for axonal regrowth and cell survival (Blesch et al. 2002). Last but not least, strategies aimed at recruiting and re-modeling endogenous NSC for tissue regeneration have become popular because they avoid detrimental immune responses, by-pass feasibility problems

Received September 11, 2009; revised manuscript received/accepted November 11, 2009.

Address correspondence and reprint requests to Rachelle Franzen, Tour de Pathologie B36, +1, 1/4A Avenue de l'Hôpital 1, 4000 SartTilman, Liège, Belgium. E-mail: rfranzen@ulg.ac.be

${ }^{1}$ These authors contributed equally to this study.

Abbreviations used: BBB, Basso, Beattie and Bresnahan; BDNF, brain-derived neurotrophic factor; BrdU, bromo-deoxyuridine; GFAP, glial fibrillar acidic protein; NSC, neural stem cell; SCI, spinal cord injury; TBS, Tris-buffered saline. 
of obtaining graftable cells and circumvent ethical issues associated with embryonic stem cells.

The exact function of adult neurogenesis in the intact mammalian CNS remains elusive. Neurogenesis has been demonstrated to be regulated by various physiological and pathological conditions, including physical exercise. In the hippocampus, Kempermann et al. (1997) found that an enriched housing environment increased the survival of newly generated neuronal cells in the dentate gyrus of adult mice. Since then, various forms of exercise have been observed to enhance hippocampal neurogenesis including voluntary running (Brown et al. 2003), forced running in a motor-driven wheel (Xu et al. 2006) or on a treadmill ( $\mathrm{Ra}$ et al. 2002), and swimming (Ra et al. 2002). Enriched environments have also been reported to increase neural progenitor cell proliferation in the sub-ventricular zone of adult rats after stroke injury (Komitova et al. 2005). Bodyweight supported treadmill training has been used for a number of years to promote the rehabilitation of spinal cord injured patients. It is thought to have an effect on the reorganization of locomotor networks along the spinal cord, generating new patterns of muscle activity (Grasso et al. 2004). Other favorable effects have been reported, like the stimulation of serotonergic fiber growth (Multon et al. 2003; Engesser-Cesar et al. 2007). Furthermore, enhanced physical activity has recently been shown to lead to increased ependymal cell proliferation (Cizkova et al. 2009).

The aim of this study was to further characterize endogenous neural stem cells in the adult rat spinal cord, their response to injury and the effect of physical exercise on their neural stem cell identity.

\section{Material and methods}

\section{Animals}

Fifty-one adult female Wistar rats (250-300 g) from the animal facility of Liege University were used for this study. Females were preferred to males, as these latter develop severe neurogenic pulmonary edema when submitted to SCI (Sedý et al. 2007). Females also develop much less urinary retention problem after injury than males. All experiments were conducted according to the ethical laws and regulations of Belgian National Fund for Scientific Research. Animals were sub-divided into four experimental groups:

1. Uninjured untrained control rats $(n=10)$.

2. Uninjured rats with daily treadmill training for 28 days $(n=8)$.

3. Spinal cord injured untrained rats, with survival times of 7 $(n=9), 14(n=8)$ and $28(n=9)$ days.

4. Spinal cord injured rats with treadmill training for 28 days $(n=7)$.

Animals were housed individually with access to water and food ad libitum. One week before the beginning of the experiments, animals were placed in the different surroundings of the behavioral tests on a daily basis, to get accustomed to them.
Spinal cord injury

Animals were anaesthetized by inhalation of Isoflurane $(2-3 \%$ Forene ${ }^{\circledR}$, Abbott, Queenborough, Kent, UK) administered at a flow rate of $1 \mathrm{~L} / \mathrm{min}$. The compression injury was performed as described by Vanicky et al. (2001). Briefly, a 2-French Fogarty arterial embolectomy catheter (Edwards Lifesciences LLC, Irvine, CA, USA) was inserted into the epidural space at T10 level and moved rostrally for two metameric levels before being inflated with a liquid volume of $15 \mu \mathrm{L}$ for $5 \mathrm{~min}$. The balloon was then deflated and carefully removed. Animals had their bladder manually emptied twice daily until recovery of spontaneous micturition occurred. Animals were carefully inspected for weight loss, dehydration and signs of autophagia. Care was taken to prevent infection by intraperitoneal antibiotics treatment (amoxicilline-clavulanic acid) during 1 week.

A $15 \mu \mathrm{L}$-lesion results in a direct complete paraplegia, followed by a progressive, spontaneous but partial functional recovery after 4 weeks. Indeed, this volume of inflation of the balloon allows the preservation of sufficient white matter to produce basic locomotion after injury (Vanicky et al. 2001), and therefore represents an interesting model to assess the efficacy of experimental strategies.

\section{Bromo-deoxyuridine treatment}

During the first 3 days following the injury, rats received intraperitoneal (i.p.) injection of bromo-deoxyuridine (BrdU, $100 \mathrm{mg} / \mathrm{kg}$; Sigma, St Louis, MO, USA) to label dividing cells.

\section{Treadmill training}

Treadmill training was performed on a custom-built device and consisted in three daily sessions of 10 min separated by 5 min-breaks (Multon et al. 2003). Treadmill speed was set at $3.5 \mathrm{~m} / \mathrm{min}$ for injured rats and $7 \mathrm{~m} / \mathrm{min}$ for uninjured rats. The first treadmill session was performed the day after the compression-injury in injured rats. Until recovery of weight support, injured animals were suspended in a harness to allow locomotion during the treadmill training, and their hind limbs mobilized manually to imitate normal stepping.

Behavioral analyses

Following SCI, locomotion, sensorimotor skills and micturition were monitored as follows:

Open field locomotor tests

Rats were observed twice a week, while moving freely in an open field. Hind-limb movement was scored and attributed by observers blinded to the treatments. The 21-point Basso, Beattie and Bresnahan (BBB) locomotor rating scale (Basso et al. 1995) and the 6-point-open field scale were applied (Gale et al. 1985; Gaviria et al. 2002). The results were analyzed using ANOvA for repeated measures.

\section{Placing response test}

This test has been reported to assess the ability of an animal to place the hind-limbs onto the surface of an obstacle following contact with the dorsal surface of the foot (Kunkel-Bagden et al. 1993; Gaviria et al. 2002). The normal response to this stimulus is to withdraw and lift the hind-limb and place the paw upon the obstacle. The score given is the mean number of normal responses to 10 stimulations. The test was performed twice a week and the results were analyzed 
using ANOVA for repeated measures followed by the post-hoc Scheffe test.

\section{Recovery of bladder function}

Animals were inspected during daily care for bladder distension and control. The post-injury day at which each animal recovered autonomous micturition was noted and the results were compared using the $T$-test for independent samples.

Histological analyses

Tissue processing

After appropriate survival delays, animals were deeply anaesthetized with pentobarbital (intraperitoneal injection of $1 \mathrm{~mL}$ of Nembutal, CEVA Santé Animale, Libourne, France) and perfused with $500 \mathrm{~mL}$ of 4\% paraformaldehyde in $0.1 \mathrm{M}$ phosphate buffer saline ( $\mathrm{pH} 7.4)$. The spinal cords were rapidly dissected out and post-fixed in the same fixative for $24 \mathrm{~h}$ at $4{ }^{\circ} \mathrm{C}$ and then kept for $48 \mathrm{~h}$ in $30 \%$ sucrose for cryoprotection. Two tissue blocks $(0.75-\mathrm{cm}$ long) were taken from both sides of the lesion epicenter. Tissue blocks were then transversely cryo-sectioned in $20 \mu \mathrm{m}$ serial sections, mounted on gelatine-coated glass slides and stored at $-20^{\circ} \mathrm{C}$ until used.

\section{Antibodies}

The primary antibodies used for immunostaining were the following: rat anti-BrdU (1/100, AbD Serotec, Düsseldorf, Germany), goat antiSOX-2 (1/500, Santa Cruz Biotechnology, Santa Cruz, CA, USA), rabbit anti-CD133 (1/100, AbCam, Cambridge, UK), mouse antinestin (1/100, Chemicon, Temecula, CA, USA), mouse anti-glial fibrillar acidic protein (GFAP, 1/500, Sigma), rabbit anti-S100B (1/ 500, DakoCytomatic, Glostrup, Denmark), rabbit anti-NG2 (1/500, Chemicon), mouse anti-CD11b (1/1000, clone OX-42, Serotec), rabbit anti-Ki67 (1/250, Novocastra, Belgium) and chicken antiBDNF (1/250, R \& D Systems, Minneapolis, MN, USA). Secondary antibodies used were fluorochrome-conjugated donkey anti-mouse, anti-rabbit, anti-goat or anti-chicken (FITC or Rhodamine conjugated, 1/500, Jackson Immuno-Research, West Grove, PA, USA).

\section{Immunofluorescence stainings}

After permeabilization in Tris-buffered saline (TBS, $\mathrm{pH} 7.6)+0.2 \%$ Triton X-100 for $15 \mathrm{~min}$, tissue sections were incubated in a blocking buffer $(1 \%$ bovine serum albumin and $3 \%$ appropriate normal serum in TBS $+0.2 \%$ Triton $\mathrm{X}-100$ ) for $1 \mathrm{~h}$, and thereafter overnight in the primary antibody diluted in the blocking buffer. After several TBSTween and TBS rinses, the sections were incubated for $2 \mathrm{~h}$ with the secondary fluorochrome-conjugated antibody (see above) diluted in TBS and $1 \%$ of appropriate normal serum, rinsed three times in TBS $+0.05 \%$ Tween 20 , and $10 \mathrm{~min}$ in TBS. Cell nuclei were labeled with Hoechst 33342 (Invitrogen Molecular Probes, Eugene, OR, USA; $0.167 \mu \mathrm{g} / \mathrm{mL}$ ) before the last rinses in distilled water. Sections were finally cover-slipped with Vectashield mounting medium (Labconsult, Brussels, Belgium). Negative controls were obtained by (i) omission of the primary antibody and (ii) incubation of the primary antibody with its specific blocking peptide (e.g. for Sox-2 labeling) before applying it on the tissue sections.

Image analysis

Sections were examined and images captured using either an Olympus AX70 microscope equipped with an Olympus DP50 digital camera and $\times 10, \times 20, \times 40$ objectives, either an Olympus Fluoview FV1000 confocal system equipped with the Olympus IX81 inverted microscope (Olympus Europa GmbH, Hamburg, Germany). For co-localization analyses, z-series of confocal images were projected to 2-D representations. Sox-2 immunoreactive nuclei were counted in predefined areas of transverse sections. A Hoechst 33342 labeling was performed to ensure that the Sox-2 staining was nuclear. The number of Sox-2 labeled ependymal cells was not counted because all ependymal cells were Sox-2 immunoreactive. All counts were done in a blinded manner and data were compared using T-test (or Mann-Whitney $U$ test when Kolmogorov-Smirnoff test for normality was significant) for independent samples. The significance level was set at $p \leq 0.05$.

\section{Results}

Ependymal cells of the normal, uninjured adult rat spinal cord express neural progenitor markers

In order to assess the progenitor nature of the ependymal cells lining the central canal of the uninjured adult rat spinal cord, we first performed Sox-2 and CD133 immunostainings on transverse sections taken at the thoracic level T8 of normal, uninjured spinal cords. As illustrated in Fig. 1, Sox-2 was expressed in the nuclei of all ependymal and subependymal cells of the central canal (Fig. 1a), but positive cells were also distributed throughout the rest of the spinal tissue, mostly within the grey matter (Fig. 1a"). Both negative and positive controls confirmed the specificity of the staining (Fig. $1 \mathrm{a}^{\prime}$ and $\mathrm{a}^{\prime \prime \prime}$ ). To further confirm the stem cell nature of ependymal cells, we also show that they express CD133, another recognized stem cell marker (Fig. 1b). In order to further characterize these cells, Sox-2/ GFAP, Sox-2/S100B, and Sox-2/NG2 double immunofluorescence were performed. We found that some Sox-2 expressing cells, located close to the central canal or in the dorsal horns, co-expressed GFAP or S100B (Fig. 1c-f), while the Sox-2 positive ependymal/sub-ependymal cells seemed to co-express only S100B, and not GFAP (Fig. 1c and d). With the exception of endothelial cells, no NG2immunoreactive cells were detected in the uninjured spinal cord (not shown).

\section{Spinal cord injury increased the number of endogenous neural progenitors in the lesion site}

Seven days after the thoracic spinal cord compression-lesion, Sox-2 immunoreactivity increased within the dorsal funiculi, i.e. in the lesion site (Fig. 2a and b). This observation has been further completed and confirmed with the 2D-based quantification of Sox-2 positive nuclei, illustrated on Fig. 2(c). To further characterize these Sox-2 expressing cells in the dorsal funiculi, we performed double immunofluorescence stainings using BrdU, CD11b and NG2 antibodies. As shown in Fig. 2(d), almost all Sox-2 immunoreactive cells co-expressed BrdU in their nuclei, 

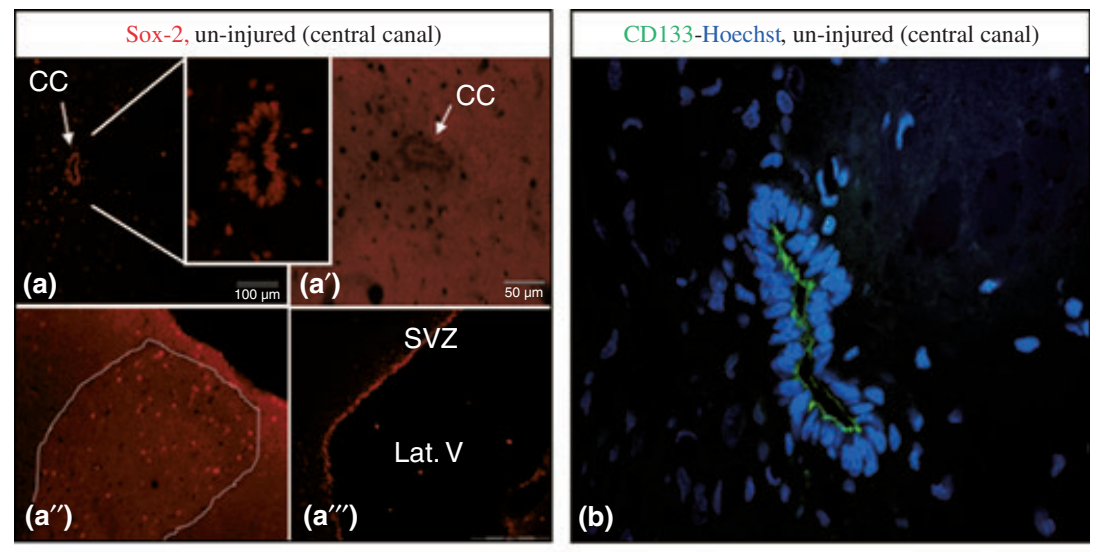

Fig. 1 SOX-2 and CD133 expression on transversal sections of uninjured spinal cord. (a) Sox-2 is expressed by all ependymal/sub-ependymal cells. (a') Negative control, where Sox-2 antibody was incubated with Sox-2 blocking peptide before use. ( $\left.a^{\prime \prime}\right)$ Sox-2 immunostained cells in the dorsal grey matter. (a"') Positive control for Sox-2 immunostaining at the subventricular zone of a rat brain. (b) CD133 immunostaining (green) of ependymal cells. (c, e) GFAP does not co-localize with Sox-2 in the ependymal cells, but close to it and also in the rest of the parenchyma (white arrows). (d, f) S100 co-localizes with Sox-2 in ependymal cells and over the parenchyma (white arrows). CC, central canal; SVZ, subventricular zone; Lat. V, lateral ventricule.
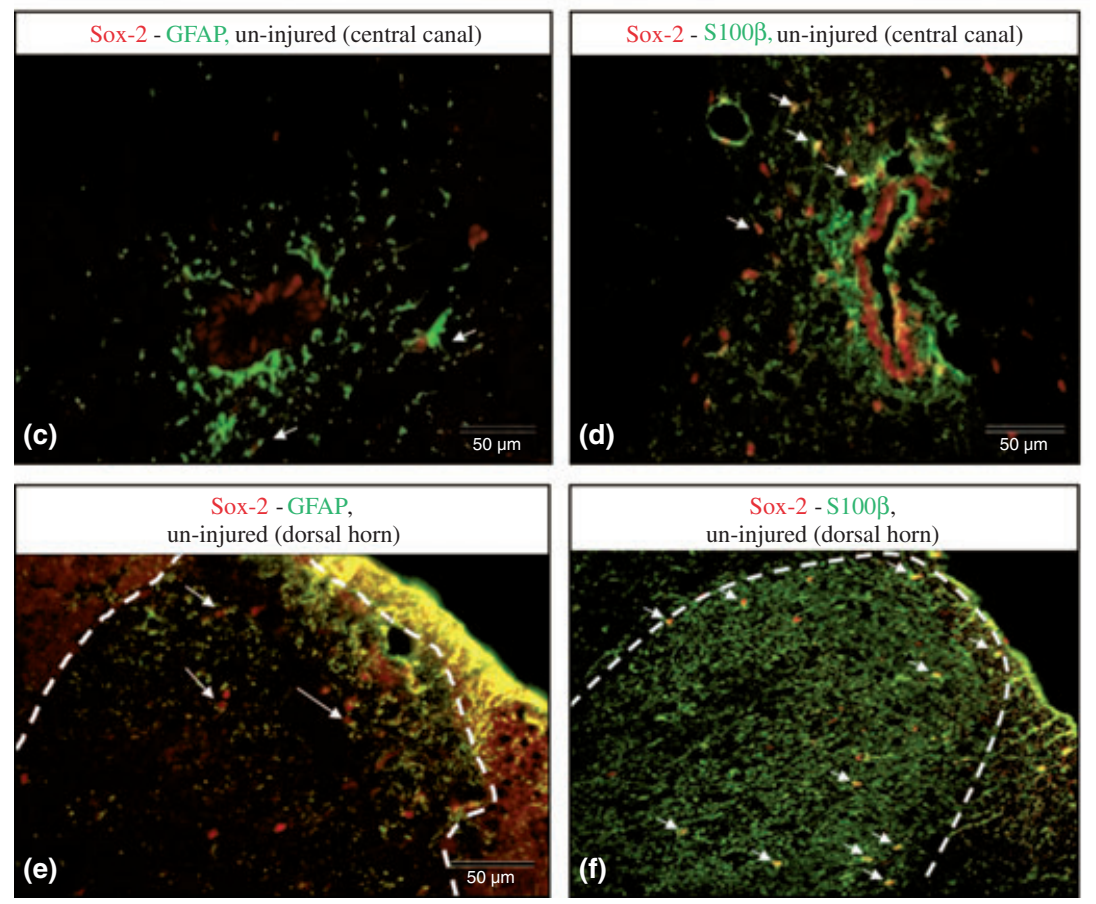

demonstrating that these cells were proliferating at the time of injury. These cells were not inflammatory cells, as no colocalization of CD11b and Sox-2 immunoreactivity could be found (Fig. 2e). Some Sox-2 cells were immunoreactive for the progenitor cell marker NG2, which contrasts with the distribution in uninjured spinal cord. These Sox-2/NG2positive cells were largely located in the lesion site and more scarcely found around the central canal (Fig. 2f-h).

Spinal cord injury induces proliferation and phenotypical changes of ependymal cells

To assess cell proliferation, both Ki67 and BrdU immunostainings were performed. SCI triggered ependymal cell proliferation, as shown in Fig. 3(a) and (b). It is interesting to note that ependymal cells still expressed Sox-2, with some of them co-expressing Ki67 (Fig. 3b). 28 days after injury, while Ki67 staining was no more detectable (data not shown), numerous BrdU-positive cells (some also expressing Sox-2) were observed both in the ependymal cells as well as in the dorsal region of the spinal cord, whereas fewer labeled cells could be observed in the ventral region, suggesting a shift of cells away from the central canal towards the lesion site (Fig. 3c). The injury also increased GFAP expression, which was expressed by ependymal Sox-2 positive cells (Fig. 3d), contrasting with the situation observed in uninjured cord. Injury also significantly induced nestin expression in ependymal cells by 7 days after injury (Fig. 3e), raising the level of nestin-staining around the central canal above the scattered incidence observed in the uninjured spinal cord (see Fig. 4a). The number of nestin-positive ependymal cells subsequently decreased over time, eventually returning to base-line levels observed in the normal 

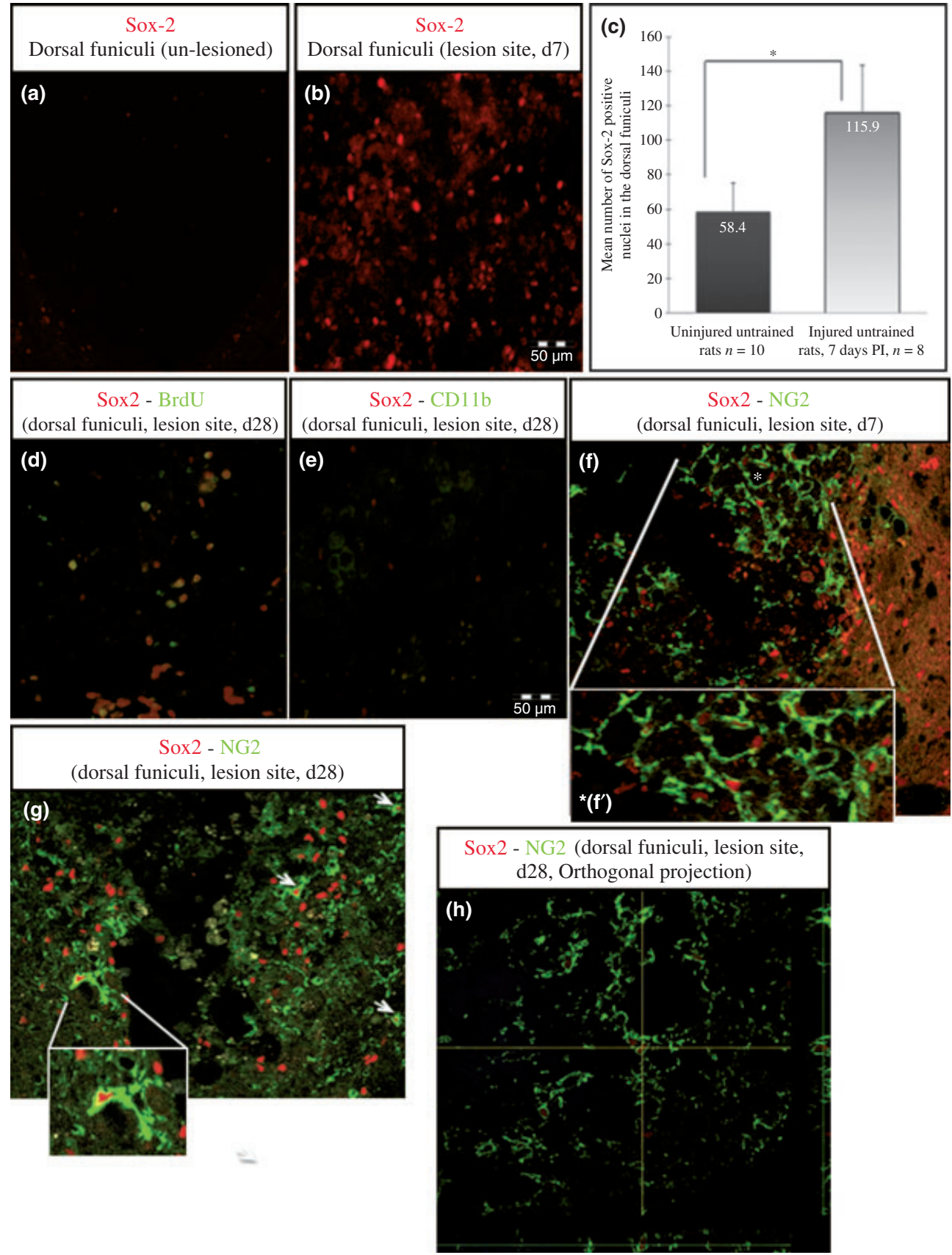

Fig. 2 SOX-2 expression on transversal sections after spinal cord injury. $(a-c)$ The number of Sox-2 immunoreactive cells in the lesion site (dorsal funiculi) increases significantly 7 days after injury $\left({ }^{*} p<0.05\right)$. These cells were proliferating at the time of injury, as shown by the co-expression of Sox-2 and BrdU (d) and are not

spinal cord. However, many nestin immunoreactive cells could still be detected in the lesioned parenchyma. These results strongly support the progenitor-type nature of the ependymal cells. It is also interesting to note that ependymal cells from the central canal do express the neurotrophic factor BDNF, 28 days after injury (Fig. 3f). inflammatory cells, as they do not co-express CD11b (e). Some Sox-2 cells within the lesion site do co-express NG-2, at both 7 and 28 days post-injury ( $f$ and $g$ ). (h) Orthogonal projection demonstrating the co-expression of NG2 and Sox-2 in the same cell within the lesion site.

Treadmill training maintains the stem nature of ependymal cells and improves functional recovery

Treadmill training for 28 days had an effect on nestin immunoreactivity in ependymal cells of both uninjured and injured spinal cords. Treadmill training, by itself, significantly increased the number of nestin immunoreactive cells 

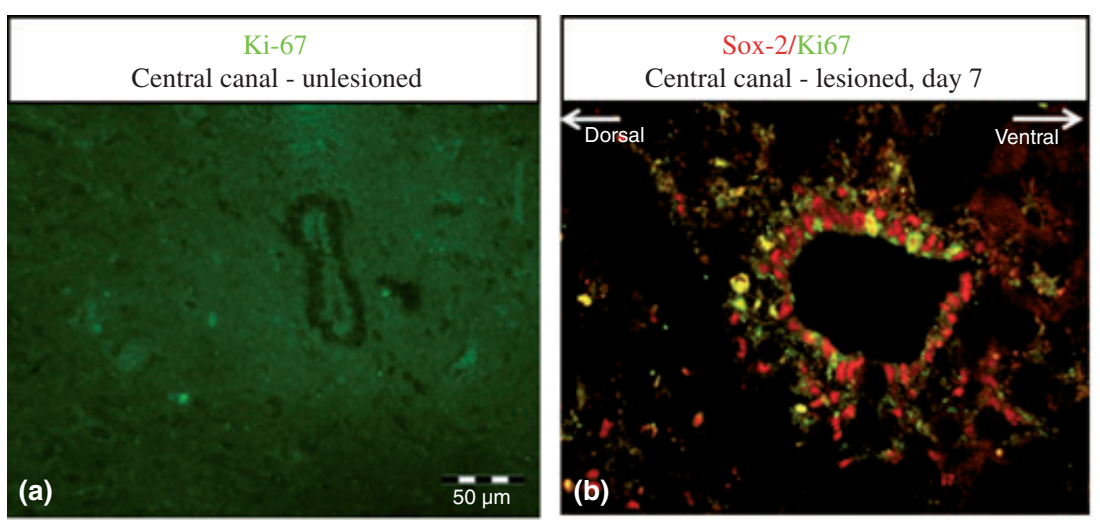

Fig. 3 Ependymal cell proliferation and plasticity after spinal cord injury. Ki67 and BrdU immunostainings of the ependymal cells reveal their proliferative phenotype after spinal cord injury. If no Ki67 could be detected in unlesioned spinal cord (a), its expression was increased 7 days after injury in the Sox-2 expressing cells (b, yellow cells). BrdU, injected during 3 days following the injury, was also incorporated by ependymal cells. BrdU cells seem to move towards the lesion site, as the majority of cells was observed in the dorsal part of the cord, where they still co-express Sox-2 (c, arrows). (d) GFAP (green)/Sox-2 (red) double immunofluorescent staining of ependymal cells, at day 7 post-injury, illustrates the phenotypical change of these cells, which didn't express GFAP in normal condition (compare to Fig. 1c). (e) Nestin immunofluorescent staining at day 7 postinjury, showing the immature phenotype adopted by ependymal cells after injury. ${ }^{*}$ = central canal. (f) BDNF immunofluorescent staining (red) of ependymal cells from the central canal $\left({ }^{*}\right), 28$ days post-injury. Specificity of the staining was controlled by omission of the first antibody $\left(f^{\prime}\right)$.
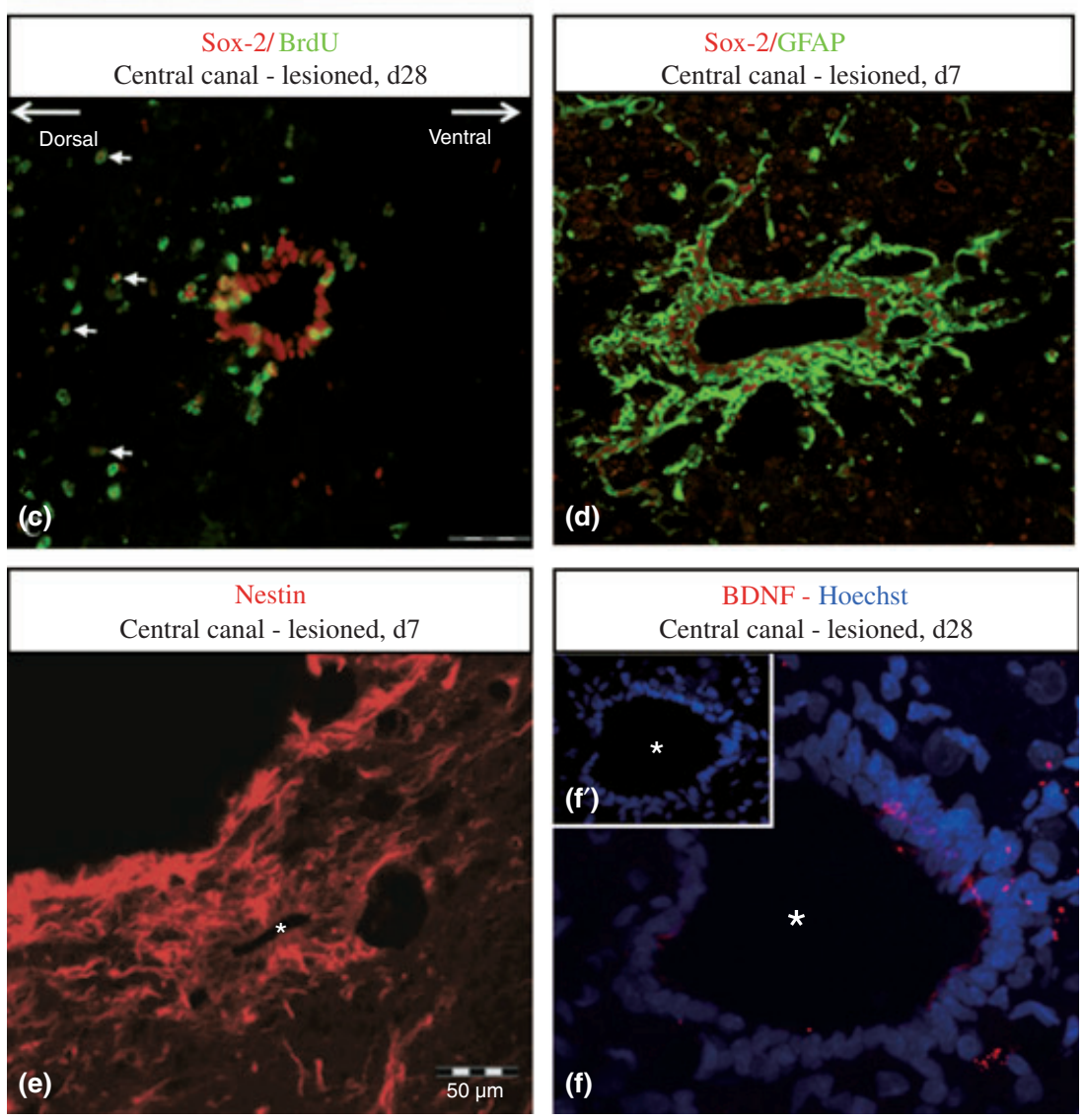

from $2.5 \pm 0.3(n=10)$ to $4.1 \pm 0.7(n=7)$ labeled cells per section $(t=2.18 ; p=0.045)$ (Fig. $4 \mathrm{a}, \mathrm{c}$ and e). Also, the injury-induced increase in the number of nestin-positive ependymal cells (which, as mentioned earlier, returned to basal levels by 28 days after injury), was partially maintained in treadmill-trained rats (Fig. 4b, d and f).

Spinal cord injured animals were submitted to three behavioral assessments (locomotion, paw placing and micturition recovery) in order to evaluate the effect of treadmill training on functional recovery. Concerning locomotor skills assessed by the two open field tests, mean scores over time were numerically higher in the treadmill-trained group, no matter which scale was used (Fig. 5a and b). The difference in values between trained and untrained animals did not reach statistical significance [ANOVA repeated measures, $F(1,13)=1.59961 ; p=0.23]$, but it became evident that the only rats capable of achieving weight support and a mean score of 9 (in the BBB test) or 3 (in the 6-point test), belonged to the trained-group. The placing response scores were also significantly improved with treadmill training [ANOVA repeated measures, $F(1,13)=11.15 ; p=0.0053$; Fig. $5 \mathrm{c}$ ], confirming the beneficial effects of physical exercising. Training was also beneficial for recovery of autonomous micturition, which occurred at post-injury day $11 \pm 1$ for the treadmill-trained rats versus day $15 \pm 1$ for untrained rats (Student's $t$-test, $t=-2.16 ; p=0.048$ ) (not shown). The 

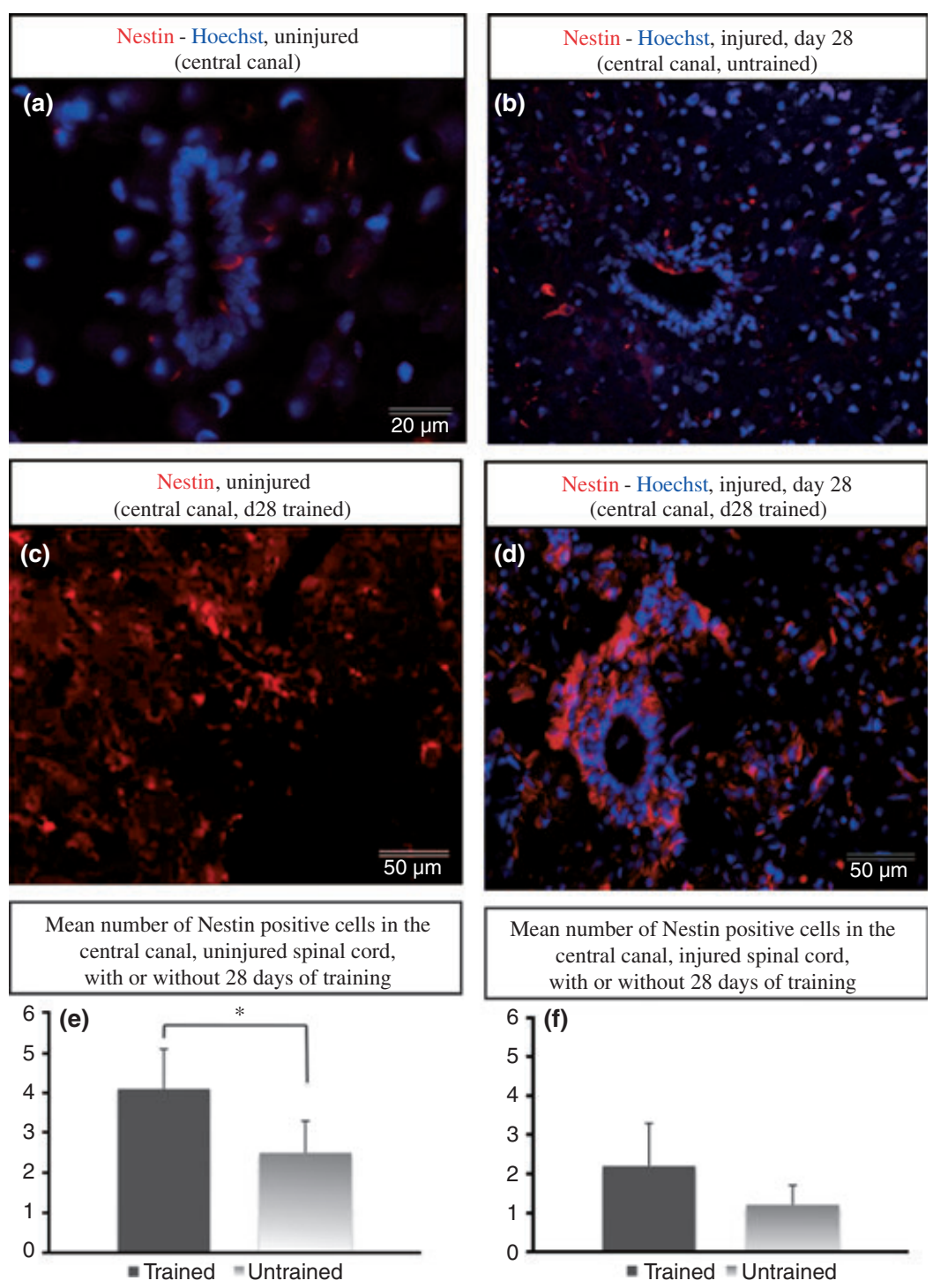

Fig. 4 Nestin expression by ependymal cells is maintained by exercise. Nestin immunofluorescent staining (red) of the central canal of uninjured spinal cord, without or with 28 days of treadmill training (a and c, respectively), and after spinal cord compression injury, without or with 28 days of treadmill training ( $b$ and $d$, respectively). Nestin, which normally decreases with time (compare Fig. 3e with $b$ ), is maintained by treadmill exercise. (e and f) Histograms illustrating the quantification of nestin expressing-cells in the ependyma, showing that chronic treadmill training increases nestin expression. ${ }^{*} p<0.05$. separation of the animals into two groups ( $n=7 /$ group), based on their BBB scores (i.e. below or above 9, reflecting the onset of weight support), revealed that more nestinimmunoreactive ependymal cells were found in the spinal cord of rats belonging to the group with a BBB score $>9$ (Mann-Whitney $U$ test, $p=0.041$ ). This suggests that neural progenitor cell recruitment may be associated with behavioral recovery (Fig. 6).

\section{Discussion}

Ependymal/sub-ependymal cells are known to be a source of endogenous neural stem cells in the adult spinal cord (Meletis et al. 2008). However, information about their phenotypical plasticity after SCI remain poorly understood, despite their relevance to prospective therapeutic intervention strategies.
The data presented here show that Sox-2 expressing cells can be found throughout the parenchyma of the normal spinal cord, particularly in the nuclei of all ependymal/subependymal cells surrounding the central canal. This is the first demonstration of the immunoreactivity for Sox-2 in the adult rat spinal cord and appears to reflect the distribution that has already been described in human spinal cord ependymal/sub-ependymal cells (Dromard et al. 2008). Sox-2 is an interesting marker, since the expression of this transcription factor has been associated with the maintenance of a neural progenitor identity, and with the inhibition of neuronal differentiation (Graham et al. 2003). The distribution of Sox-2 immunoreactivity in the spinal cord is in accordance with that observed in the brain: Sox-2 immunoreactive cells were also being located in neurogenic regions as well as throughout the whole brain parenchyma (Komitova and Eriksson 2004). The finding of a widespread 
Fig. 5 Effect of treadmill training on locomotor recovery. Both scales (BBB and 6point open-field, respectively $a$ and $b$ ) show the same recovery evolution, with trained rats reaching higher scores than untrained ones, corresponding to weight-supporting steps for trained rats and plantar placement without weight-support for untrained ones (dashed lines). Placing response test was significantly improved by training (c). ${ }^{*} p<0.01$.

distribution of Sox-2 immunoreactive cells over the spinal cord parenchyma agrees with previous studies showing that a substantial number of neural progenitors can be identified in the spinal tissue of adult rats [Yamamoto et al. 2001; Horner et al. 2000; Horky et al. 2006). To further characterize these Sox-2-positive cells, we performed a double staining with GFAP and S100B and showed that in uninjured spinal cords, Sox-2-positive cells in the ependymal lining co-expressed $\mathrm{S} 100 \mathrm{~B}$, but not GFAP (which is expressed by tanicytes but not ependymocytes), while Sox-2 cells in the rest of the spinal cord tissue co-expressed both S100B and GFAP. In the adult brain, double stained Sox-2/GFAP cells are essentially found in non-neurogenic areas, and very rarely in neurogenic
Rating scale:

0. No observable hindlimb movement !

8. Sweeping or plantar placement of the paw without weight support 9. Plantar placement of the paw with weight support in stance only or occasional OR Consistent weight supported dorsal stepping and no plantar stepping ,

21. Normal walking.

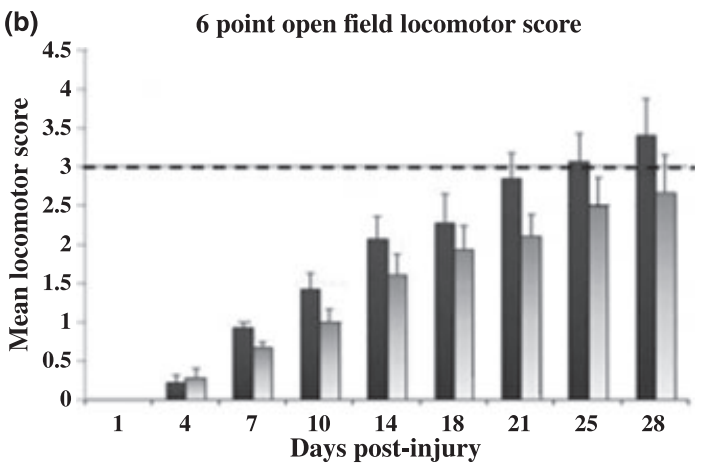

Rating scale:

0. No movement of hindlimbs, no weightbearing

1. Barely perceptible movement of hindlimbs, no weight-bearing

2. Frequent and/or vigorous movement of hindlimbs, no weight-bearing

3. Hindlimbs can partially support weight, may make few steps

4. Total weight

support, walking with major deficit

5. Walking with mild deficit 6. Normal walking

Treadmill trained rats $n=7 \quad$ Untrained rats $n=9$

(c)

Placing response test

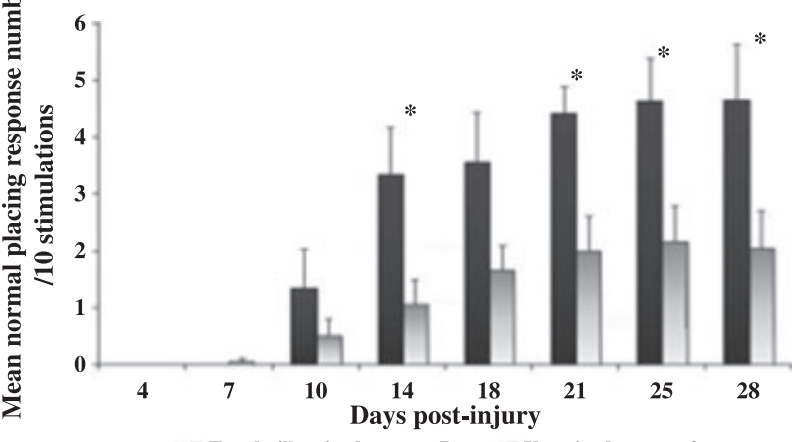

- Treadmill trained rats $n=7 \quad$ Untrained rats $n=9$

regions like the sub-ventricular and sub-granular zones (Komitova et al. 2005). Our data in the adult spinal cord are in accordance with these findings in the brain: nearly all Sox-2 positive cells in the spinal tissue seem to be astroglia, with the notable exception of Sox-2 ependymal cells which were GFAP negative. Our results are also in line with the finding that $\mathrm{S} 100 \mathrm{~B}$ is expressed by ependymal cells (Steiner et al. 2007).

After injury, the immunoreactivity for Sox-2 significantly increased in the lesion site (dorsal funiculi). A 3D-based stereological quantification method was not used as our goal was not to obtain an absolute number of Sox-2 expressing cells within the lesion site, but to better illustrate the 


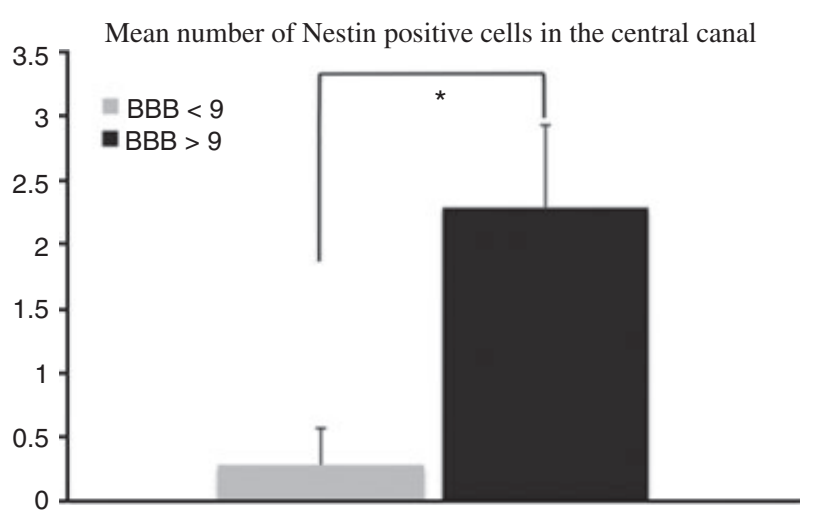

Fig. 6 Increased mobility is correlated to immature phenotype of ependymal cells. Histogram showing the mean number of nestin expressing cells in the ependymal canal from rats with BBB locomotor scores $<9$ (no weight-supported steps, $n=7$ ) and rats with locomotor scores $>9$ (weight-supported steps, $n=7$ ), 28 days post-injury. The graph demonstrates the significant correlation between an increased mobility of the rats and the number of nestin expressing cells in vivo. ${ }^{*} p<0.05$.

increased immunoreactivity observed 7 days after injury. Also, when 2D- and 3D-based quantification methods were compared, no significant differences were pointed out (Baquet et al. 2009). The majority of these Sox-2 cells were mitotically active at the time of injury, as indicated by the nuclear incorporation of BrdU. Spinal cord ependymal cells also proliferated in response to injury, in agreement with previous publications (Cizkova et al. 2009; Mothe and Tator 2005). The heterogeneous distribution of Sox-2/BrdUpositive cells within the spinal cord might reflect the migration of ependymal cell towards- and their accumulation within the lesion site. The co-expression of Sox-2 and NG2 markers in the lesion site is in accordance with Horky and colleagues, who described a second immature population of stem cells originating in the sub-ependymal region, and which exhibited massive proliferation and preferential differentiation into NG2-expressing glia. Our Sox-2/NG2 positive cells might therefore represent the population of quiescent endogenous stem/progenitor population that divides early after injury, and replaces the vulnerable population of active progenitor population existing in the spinal cord (Horky et al. 2006). The double labeling for Sox2 and GFAP in ependymal cells as early as 7 days post-injury was also described by Horky (Horky et al. 2006). According to Doetsch's work, GFAP-positive cells might not only indicate fully-differentiated glial cells, but also immature astrocytes that can act as neural stem cells (Doetsch et al. 1999).

Our data also strongly suggest a beneficial effect of exercising on neural stem cell mobilization and behavioral recovery after SCI. The BBB and open-field scores revealed differences between trained and untrained groups, but the values did not reach the level of significance. This can be explained by the fact that both scores are not linear scales, ant that a 1-point increase can as well reflect a significant functional improvement (as the weight-support level of stepping) as a simple difference in stepping coordination frequency, which is functionally less essential. It is therefore more appropriate to compare the level of functional recovery, and to conclude that in our study, only trained rats reached the level of weight-supported steps. The improvement in functional recovery could be explained by an increased maintenance of the stem-type nature of the ependymal cells, as we found a positive relation between behavioral locomotor scores and the number of nestin expressing cells around the central canal. Similar observations have been made in the hippocampus of adult rats, where the number of proliferating astrocytes and neuroblasts in the sub-granular zone of the dentate gyrus increased after chronic treadmill (Uda et al. 2006). Improved behavioral scores and elevated neurogenesis in the sub-granular zone of the dentate gyrus have also been promoted by enforced physical training after focal cerebral ischemia (Lee et al. 2008). A correlation between increased locomotor scores and proliferative cells in the injured spinal cord was also described by Takahashi and coworkers (Takahashi et al. 2003). The potential roles of NSC in physical activity-mediated functional recovery after SCI have been discussed recently by Teng et al. (2006). Our results, and more particularly the demonstration of nestin and BDNF expression in ependymal cells, support their hypothesis that endogenous NSC may mediate the functional recovery noted in SCI following physical activity-based treatments. Exercise stimulates endogenous neural stem cells to proliferate and produce neurotrophic factors, such as BDNF and glial cell line-derived neurotrophic factor, which in turn mediate anatomical plasticity resulting in improved motor function (Teng et al. 2006). BDNF has recently been shown to play an important role in mediating the beneficial effects of exercise on the recovery of locomotion after SCI (Ying et al. 2008), and also to enhance the proliferation rate of nestin-positive and BrdU-positive neural stem cells in vitro (Islam et al. 2009). In addition, the maintenance of nestin expression could also be explained by the recent demonstration of the binding of the transcription factor Sox-2 to the nestin enhancer (Miyagi et al. 2006) and the recruitment of Sox-2 to maintain nestin expression in neural progenitor cells (Jin et al. 2009).

To summarize, our results confirm that ependymal cells from the adult rat spinal cord exhibit progenitor-like characteristics. We also show that these cells can be recruited not only by a spinal cord compression-injury, but interestingly by physical exercising, i.e. treadmill training, which improves locomotor recovery after a spinal cord compression-injury. Interestingly, this behavioral improvement is linked to an increased number of nestin immunoreactive ependymal cells. Although some animals displaying higher 
locomotor scores were not in the treadmill-trained group (though most were), there was a clear relationship between increased mobility and the recruitment of progenitors. The combined strategy of increased physical exercise and the manipulation of the spinal cord microenvironment to maximize endogenous stem cell's contribution to spinal cord repair represents a potential therapeutic option in the future.

\section{Acknowledgements}

The authors are grateful to Ms Jeanine Mosen, Ms. Coralie DiPrima and Ms. Murielle Wouters for their expert technical assistance to this work. This work was supported by grant 3.4.563.04 of the National Fund for Scientific Research (Belgium) to JS, the Specific Targeted Research Project 'RESCUE' from the 6th Framework Program of the European Community and the 'Gunter Verbraeckel Foundation for scientific research on spinal cord injury' (private foundation, Belgium). RF was research associate of the National Fund for Scientific Research (FNRS-Belgium) until end of 2007.

\section{References}

Baquet Z. C., Williams D., Brody J. and Smeyne R. J. (2009) A comparison of model-based (2D) and design-based (3D) stereological methods for estimating cell number in the substantia nigra pars compacta (SNpc) of the C57BL/6J mouse. Neuroscience 161, 1082-1090.

Basso D. M., Beattie M. S. and Bresnahan J. C. (1995) A sensitive and reliable locomotor rating scale for open field testing in rats. J. Neurotrauma 12, 1-21.

Blesch A., Lu P. and Tuszynski M. H. (2002) Neurotrophic factors, gene therapy, and neural stem cells for spinal cord repair. Brain Res. Bull. 57, 833-838.

Brown J., Cooper-Kuhn C. M., Kempermann G., Van Praag H., Winkler J., Gaga F. H. and Kuhn H. G. (2003) Enriched environment and physical activity stimulate hippocampal but not olfactory bulb neurogenesis. Eur. J. Neurosci. 17, 2042-2046.

Cizkova D., Nagyova M., Slovinska L. et al. (2009) Response of ependymal progenitors to SCI or enhanced physical activity in adult rat. Cell. Mol. Neurobiol. 29, 999-1013.

Doetsch F., Caillé I., Lim D. A., Garcia-Verdugo J. M. and AlvarezBuylla A. (1999) Subventricular zone astrocytes are neural stem cells in the adult mammalian brain. Cell 97, 703-716.

Dromard C., Guillon H., Rigau V. et al. (2008) Adult human spinal cord harbors neural precursor cells that generate neurons and glial cells in vitro. J. Neurosci. Res. 86, 1916-1926.

Engesser-Cesar C., Ichiyama R. M., Nefas A. L., Hill M. A., Edgerton V. R., Cotman C. W. and Anderson A. J. (2007) Wheel running following SCI improves locomotor recovery and stimulates serotonergic fiber growth. Eur. J. Neurosci. 25, 1931-1939.

Gale K., Kerasidis H. and Wrathall J. R. (1985) Spinal cord contusion in the rat: behavioral analysis of functional neurologic impairment. Exp. Neurol. 88, 123-134.

Gaviria M., Haton H., Sandillon F. and Privat A. (2002) A mouse model of acute ischemic spinal cord injury. J. Neurotrauma 19, 205-221.

Graham V., Khudyakov J., Ellis P. and Pevny L. (2003) SOX2 functions to maintain neural progenitor identity. Neuron 39, 749-765.

Grasso R., Ivanenko Y. P., Zago M., Molinari M., Scivoletto G., Castellano V., Macellari V. and Lacquaniti F. (2004) Distributed plasticity of locomotor pattern generators in spinal cord injured patients. Brain 127, 1019-1034.
Horky L. L., Galimi F., Gage F. H. and Horner P. J. (2006) Fate of endogenous stem/progenitor cells following spinal cord injury. J. Comp. Neurol. 498, 525-538.

Horner P. J., Power A. E., Kempermann G., Kuhn H. G., Palmer T. D., Winkler J., Thal L. J. and Gage F. H. (2000) Proliferation and differentiation of progenitor cells throughout the intact adult rat spinal cord. J. Neurosci. 20, 2218-2228.

Islam O., Loo T. X. and Heese K. (2009) Brain-derived neurotrophic factor (BDNF) has proliferative effects on neural stem cells through the truncated TRK-B receptor, MAP kinase, AKT, and STAT-3 signaling pathways. Curr. Neurovasc. Res. 6, 42-53.

Jin Z., Liu L., Bian W., Chen Y., Xu G., Cheng L. and Jing N. (2009) Different transcription factors regulate nestin gene expression during P19 cell neural differentiation and central nervous system development. J. Biol. Chem. 284, 8160-8173.

Johansson C. B., Momma S., Clarke D. L., Risling M., Lendhal U. and Frisén J. (1999) Identification of a neural stem cell in the adult mammalian central nervous system. Cell 96, 25-34.

Kempermann G., Kuhn H. G. and Gage F. H. (1997) More hippocampal neurons in adult mice living in an enriched environment. Nature 386, 493-495.

Komitova M. and Eriksson P. S. (2004) Sox-2 is expressed by neural progenitors and astroglia in the adult rat brain. Neurosci. Lett. 369 , 24-27.

Komitova M., Mattsson B., Johansson B. B. and Eriksson P. S. (2005) Enriched environment increases neural stem/progenitor cell proliferation and neurogenesis in the subventricular zone of strokelesioned adult rats. Stroke 36, 1278-1282.

Kunkel-Bagden E., Dai H. N. and Bregman B. S. (1993) Methods to assess the development and recovery of locomotor function after spinal cord injury in rats. Exp. Neurol. 119, 153-164.

Lee S. H., Kim Y. H., Kim Y. J. and Yoon B. W. (2008) Enforced physical training promotes neurogenesis in the subgranular zone after focal cerebral ischemia. J. Neurol. Sci. 269, 54-61.

Meletis K., Barnabé-Heider F., Carlén M., Evergren E., Tomilin N., Shupliakov O. and Frisén J. (2008) Spinal cord injury reveals multilineage differentiation of ependymal cells. PLoS Biol. 6, 1494-1507.

Miyagi S., Nishimoto M., Saito T., Ninomiya M., Sawamoto K., Okano H., Muramatsu M., Oguro H., Iwama A. and Okuda A. (2006) The Sox 2 regulatory region 2 functions as a neural stem cell-specific enhancer in the telencephalon. J. Biol. Chem. 281, 13374-13381.

Mothe A. J. and Tator C. H. (2005) Proliferation, migration, and differentiation of endogenous ependymal region stem/progenitor cells following minimal spinal cord injury in the adult rat. Neuroscience 131, 177-187.

Multon S., Franzen R., Poirrier A. L. and Schoenen J. (2003) The effect of treadmill training on motor recovery after a partial spinal cord compression-injury in the adult rat. J. Neurotrauma 20, 699706.

Ohori Y., Yamamoto S., Nagao M., Sugimori M., Yamamoto N., Nakamura K. and Nakafuku M. (2006) Growth factor treatment and genetic manipulation stimulate neurogenesis and oligodendrogenesis by endogenous neural progenitors in the injured adult spinal cord. J. Neurosci. 26, 11948-11960.

Parr A. M., Kulbatski I. and Tator C. (2007) Transplantation of adult rat spinal cord stem/progenitor cells for spinal cord injury. J. Neurotrauma 24, 835-845.

Ra S. M., Kim H., Jang M. H., Shin M. C., Lee T. H., Lim B. V., Kim C. J., Kim E. H., Kim K. M. and Kim S. S. (2002) Treadmill running and swimming increase cell proliferation in the hippocampal dentate gyrus of rats. Neurosci. Lett. 333, 123-126.

Ramon Cajal S. (1928) Degeneration and Regeneration of the Nervous System. Hafner, New York. 
Reynolds B. A. and Weiss S. (1992) Generation of neurons and astrocytes from isolated cells of the adult mammalian central nervous system. Science 255, 1707-1710.

Sedý J., Urdzíková L., Likavcanová K., Hejcl A., Jendelová P. and Syková E. (2007) A new model of severe neurogenic pulmonary edema in spinal cord injured rat. Neurosci. Lett. 423, 167-171.

Shihabuddin L. S. (2008) Adult rodent spinal cord-derived neural stem cells: isolation and characterization. Methods Mol. Biol. 438, 55-66.

Steiner J., Bernstein H. G., Bielau H., Berndt A., Brisch R., Mawrin C., Keilhoff G. and Bogerts B. (2007) Evidence for a wide extra-astrocytic distribution of S100B in human brain. BMC Neurosci. 8, 2

Takahashi M., Arai Y., Kurosawa H., Sueyoshi N. and Shirai S. (2003) Ependymal cell reactions in spinal cord segments after compression injury in adult rats. J. Neuropathol. Exp. Neurol. 62, 185-194.

Teng Y. D., Liao W. L., Choi H., Konya D., Sabharwal S., Langer R., Sidman R. L., Snyder E. Y. and Frontera W. R. (2006) Physical activity-mediated functional recovery after spinal cord injury: potential roles of neural stem cells. Regen. Med. 1, 763-776.

Uda M., Ishido M., Kami K. and Masuhara M. (2006) Effects of chronic treadmill running on neurogenesis in the dentate gyrus of the hippocampus of adult rat. Brain Res. 1104, 64-72.
Vanicky I., Urdzikova L., Saganova K., Ciskova D. and Galik J. (2001) Simple and reproducible model of spinal cord injury induced by epidural balloon inflation in the rat. J. Neurotrauma 18, 13991407

Weiss S., Dunne C., Hewson J., Wohl C., Wheatley M., Peterson A. C. and Reynolds B. A. (1996) Multipotent CNS stem cells are present in the adult mammalian spinal cord and ventricular neuroaxis. J. Neurosci. 16, 7599-7609.

Xu W. P., Shan L. D., Gong S., Chen L., Zhang Y. J., Yin Q. Z., Hisamitsu T., Jiang X. H. and Guo S. Y. (2006) Forced running enhances neurogenesis in the hippocampal dentate gyrus of adult rats and improves learning ability. Sheng Li Xue Bao 58, 415420.

Yamamoto S., Yamamoto N., Kitamura T., Nakamura K. and Nakafuku M. (2001) Proliferation of parenchymal neural progenitors in response to injury in the adult rat spinal cord. Exp. Neurol. 172, 115127.

Ying Z., Roy R. R., Zhong H., Zdunowski S., Edgerton V. R. and Gomez-Pinilla F. (2008) BDNF-exercise interactions in the recovery of symmetrical stepping after a cervical hemisection in rats. Neuroscience 155, 1070-1078. 